\title{
Physical Activity and Cancer
}

\author{
Jessica Clague and Leslie Bernstein ${ }^{\dagger}$ \\ Division of Cancer Etiology, Department of Population Sciences, Beckman Research Institute, \\ City of Hope, Duarte, CA
}

\begin{abstract}
Epidemiological evidence continues to accumulate on the benefits of physical activity in relation to cancer risk, progression and mortality. Recent studies suggest that sedentary behavior may independently affect cancer risk; they also focus on factors that may explain associations with physical activity, including cancer risk factors and whether associations exist for precancerous lesions. Despite enormous efforts to examine associations between physical activity and cancer, the literature is hindered by inconsistent assessment of physical activity across studies, and incomplete consideration of variation of effects across population subgroups (for example, defined by body size, age or sex) or tumors subgroups (organ location, receptor status, or molecular subtype), and whether other factors explain study results. Clearly, public health recommendations for appropriate changes in activity levels are needed; unfortunately, at this time, we have no exact physical activity prescription to give to the public.
\end{abstract}

\section{Keywords}

Review; Physical activity; Cancer risk; Cancer survival; Recreational activity; Occupational activity; Exercise; Biological mechanisms; Methodology; Sedentary behavior; Sitting time; Breast cancer; Colon cancer

\section{Introduction}

The health benefits of participating in regular physical activity are well-documented, and include reductions in risk of cardiovascular disease, diabetes, osteoporosis, obesity, depression, fatigue, and overall mortality [1].Compelling evidence exists for inverse relationships between physical activity and risk of breast and colon cancers [2]. For these cancers, physical activity represents one of the few modifiable risk factors that can be recommended for risk reduction [2]. Evidence suggests that physical activity may also reduce the risk of endometrial and ovarian cancer, and may possibly reduce the risk of prostate and lung cancer $[3,4]$.

Case-control and cohort studies have been the mainstay of study designs to examine associations between physical activity and cancer. Methods for data collection physical activity vary on approach (interview, self completed questionnaire, or occupational energy expenditure), periods of life covered, and whether key elements (duration of each physical activity episode, frequency of episodes, and intensity of activity) are available. It is also

\footnotetext{
${ }^{\dagger}$ Corresponding author: Leslie Bernstein, PhD, Professor and Director, Division of Cancer Etiology, Department of Population Sciences, Dean for Faculty Affairs, Beckman Research Institute, City of Hope, 1500 East Duarte Road, Duarte, CA 91010, (626) 471-7315, (626) 471-7308 (Fax), lbernstein@coh.org.
}

Disclosure

No potential conflicts of interest relevant to this article were reported. 
pertinent to distinguish between broad types of physical activity: recreational (exercise activity), occupational activity (including childcare and household activity), and transportation activity (walking or biking to work). Many studies estimate metabolic equivalents (MET)-hours of energy expenditure, a composite measure that captures intensity, frequency and duration of activity, using standard values specific to each activity and multiplying these values by the number of hours spent in the specific activity per week [5].

A number of meta-analyses have been published recently; these combine effect estimates from a series of studies to provide summary estimates of cancer risk. Pooled analyses derive similar estimates of risk, but use the original data from the studies. A major challenge in combining results across studies of physical activity and cancer is that methods of quantifying physical activity vary. Most meta-analyses compare the group with the most physical activity (however defined) to that with the least activity (sometimes inactive individuals; other times those with less than some predefined amount of activity). Further, some studies examine recent activity, whereas others evaluate measures of long-term or lifetime activity; some include only recreational activity, whereas others focus on occupational activity or combine recreational and occupational activities. Therefore, one must interpret results of these studies cautiously.

Understanding the biological mechanisms underlying the associations between physical activity and cancer will provide needed insights that permit appropriate physical activity recommendations for reducing cancer risk. A number of plausible candidate mechanisms have been proposed, some specific to one particular cancer; however the single mechanism or group of mechanisms that explains the associations between physical activity and lower cancer risk have yet to be established. For colon cancer, the major hypothesis is that physical activity lowers fecal bile acid concentrations and decreases gastrointestinal transit time. For female breast cancer, physical activity alters endogenous production of sex steroid hormones by altering menstrual cycle patterns (resulting in luteal phase defects, oligomenorrhea or secondary amenorrhea) through its impact on the hypothalamic pituitary axis and, among postmenopausal women, by controlling body weight [6•]. These mechanisms, which regulate sex steroid hormones, are also potential mediators of the associations between physical activity and endometrial and ovarian cancers. Other mechanisms have been proposed that have more generalized anticancer impact and may explain associations between physical activity and several cancer sites; these include heightening immune surveillance, reducing inflammation, increasing endogenous antioxidant enzyme systems, increasing insulin sensitivity, controlling growth factor production and activation, decreasing obesity and central adiposity, optimizing DNA repair capacity, and reducing oxidative stress [6•]. It is highly plausible that several of these mechanisms act simultaneously, and that they interact synergistically to mediate the associations between physical activity and cancer.

This report provides a review of epidemiologic studies published between January 2011 and April 2012 on the associations between physical activity and cancer.

\section{Colorectal Cancer}

\section{Colorectal Cancer Development}

An inverse association between physical activity and colon cancer risk has been consistently observed among epidemiologic studies; however, the evidence for rectal cancer remains inconclusive [7, 8]. Historically, comprehensive reviews have estimated that physical activity may reduce colon cancer risk by 20-25\% when comparing individuals with the highest levels of physical activity to those with the lowest levels [9]. Risk reductions are 
greater for case-control studies (24\%) than for cohort studies $(17 \%)$, and risk reductions are similar for occupational activity $(22 \%)$ and recreational activity (23\%) [9]. In cohort studies, colon cancer risk reduction associated with physical activity is greater for men than for women, although case-control studies suggest similar benefits for men and women [10].

Whether physical activity preferentially protects against distal or proximal colon cancer is uncertain [11]. Boyle and colleagues studied 870 colorectal cancer cases (284 proximal, 268 distal and 318 rectal) and 996 age- matched and sex-matched healthy controls, examining the timing and intensity of recreational physical activity on subsite-specific colon cancer risk [12•]. Stronger associations were observed for distal tumors than for proximal tumors. Consistent vigorous recreational physical activity ( $\geq 6 \mathrm{MET}$-hours/week) during adult years was associated with $41 \%$ lower distal colon cancer risk [95\% confidence interval (CI), $0.36-0.96]$. The association between vigorous recreational physical activity and distal colon cancer risk was limited to activity performed after age 51 years, with risk reductions of $49 \%$ for 6 to 18 MET-hours per week and $55 \%$ for 18 or more MET-hours per week. Boyle et al. did not observe any association for proximal colon cancer [odds ratio (OR) $=0.93 ; 95 \% \mathrm{CI}$, 0.59-1.45] [12•]. Although the majority of previous studies [9] have not found an association between physical activity and rectal cancer, consistent vigorous recreational physical activity averaging at least 6 MET-hours per week during adult years was associated with a reduced rectal cancer risk in one study $(\mathrm{OR}=0.62,95 \% \mathrm{CI}, 0.39-0.98)[12 \bullet]$.

Sedentary behavior, defined by prolonged sitting and low energy expenditure activities (MET-value < 1.5) has been independently associated with increased risk of several cancers [13]; however, it is still unclear whether sedentary occupations modify colon cancer risk. Boyle and colleagues et al. also investigated the effect of long-term sedentary occupational work, defined by the US Department of Labor's Dictionary of Occupational Titles Demands Strength Rating, in their case-control study [14]. Participants who reported at least 10 years of sedentary work had almost two-times greater risk of distal colon cancer $(\mathrm{OR}=1.94,95 \%$ CI, 1.28-2.93) than those who never held a sedentary job [14]. Importantly, this association was independent of the amount of recreational physical activity reported by study participants [14].

Given that body mass index [BMI, generally measured as weight $(\mathrm{kg}) / \mathrm{height}\left(\mathrm{m}^{2}\right)$ ] and physical activity are both risk factors for colon cancer, several analytic approaches have been employed to tease apart their joint and independent effects on colon cancer risk. The impact of occupational (longest-held and last job) and baseline recreational physical activity on the association between body size (trouser/skirt size) and colorectal cancer risk were investigated among members in the Netherlands Cohort Study (2,316 colorectal cases and 3,197 healthy cohort members) using a case-cohort analytic approach [15]. For women who were less likely to hold jobs than men, only recreational physical activity was considered. Low physical activity was associated with increased colorectal cancer risk, consistent with previous studies. In this study, the joint effects of physical activity and body size were assessed by comparing extremes. Smaller women (trouser/skirt size $\leq 44$, comparable to a size 14 in the US) in the highest recreational activity category (> $90 \mathrm{~min} /$ day) were compared to larger women (trouser/skirt size $>44$ ) in the lowest recreational activity category (<30 min/day); risk for each subsite of colorectal cancer was increased [Distal: hazard ratio $(\mathrm{HR})=1.95,95 \% \mathrm{CI}, 1.21-3.17$; Proximal: $\mathrm{HR}=1.70,95 \% \mathrm{CI}, 1.08-2.67$; Rectal: $\mathrm{HR}=2.56,95 \% \mathrm{CI}, 1.36-4.79]$. However, the interaction between physical activity and body size was statistically significant only for proximal tumors. Among men, the combination low occupational-plus-recreational physical activity $(<8 \mathrm{~kJ} /$ minute $)$ was associated with a $63 \%$ increased risk (95\% CI, 1.03-2.56) of distal colon cancer among men whose trouser size was $\leq 52$ (median of the cohort). No association was observed for proximal colon cancer or rectal cancer. 
Genomic instability is known to drive several carcinogenic pathways [16]. Epigenetic silencing of gene expression, primarily mediated by aberrant DNA methylation, has been implicated for colon cancer; however, its role in modifying the association between colon cancer and physical activity is not well understood [16]. Within a subset of the Netherlands Cohort Study population (603 colorectal cancer cases and 4,631 controls), Hughes and colleagues investigated the combined effects on colon cancer risk of $\mathrm{CpG}$ island methylator phenotype (CIMP) and physical activity [17]. Combined occupational-plus-recreational physical activity at baseline was associated with decreased colorectal cancer risk, regardless of molecular subtype (CIMP+/non-CIMP) ( $P$-heterogeneity=0.33)[17]. Among CIMP+ tumors, a $50 \%$ lower colorectal cancer risk $(95 \% \mathrm{CI}, 0.30-0.82)$ was observed for men and women in the intermediate activity category compared to those in the low physical activity; however, high activity was not associated with risk (HR=0.82, $95 \%$ CI, 0.49-1.38) [17]. For non-CIMP tumors, high and intermediate levels of physical activity were not associated with colorectal cancer risk (High: HR=0.69, $95 \%$ CI, 0.47-1.01; Intermediate: HR=0.81, $95 \%$ CI, 0.61-1.07) [17]. The study lacked the statistical power to stratify by tumor subsite (proximal and distal colon cancer and rectal cancer).

An emphasis has been made on trying to identify risk factors for colon adenomas, which are considered a precursor lesion for colon cancer that is detected and removed during colonoscopy or sigmoidoscopy. Wolin et al. conducted a meta-analysis of twenty studies that investigated the association between recreational physical activity and colon adenomas published through April 2010 [18•]. An inverse relationship with a $19 \%$ reduction in adenoma risk among men (95\% CI, 0.67-0.98) and a $13 \%$ risk reduction among women (95\% CI, 0.74-1.02) [18•] was observed. Among men and women combined, the association was strongest, for large/advanced polyps (OR=0.30; $95 \% \mathrm{CI}, 0.56-0.88)$ [18•].

\section{Colorectal Cancer Survival}

Physical activity before as well as after colorectal cancer diagnosis is associated with improved survival $[19 \bullet, 20 \bullet$. One study examined whether CTNNB1 (beta-catenin), which is known to play a critical role in colorectal carcinogenesis and metabolic diseases, modifies the association between physical activity and colorectal cancer survival among participants in two prospective cohort studies (the Nurses Health Study and the Health Professionals Follow-up Study) conducted in the US [21]. Colorectal cancer-specific mortality was inversely associated with increasing levels of post-diagnosis physical activity among patients whose tumors were CTNNB1-negative ( $\mathrm{HR}=0.33$, $95 \% \mathrm{CI}, 0.13-0.81$ comparing $\geq$ 18 vs. $<18$ MET-hours/week), but not among patients with CTNNB1-positive colorectal cancers [21].

\section{Breast Cancer}

\section{Breast Cancer Development}

The evidence for an association between physical activity and breast cancer has been classified as convincing [2]. Results from case-control studies and cohort studies have shown that invasive breast cancer risk is reduced by $20-40 \%$ among physically active women [2]. One of the earliest studies, a case-control study of women 40 years or younger, showed a dramatic reduction in risk (approximately 50\%) among women who averaged about 4 hours of activity per week during their reproductive years [22]. Similarly, among postmenopausal women, those with higher amounts of recreational physical activity during their lifetimes have been shown to have lower breast cancer risk [23, 24].

Epidemiologists require that a risk factor demonstrate consistency across populations before considering it as "accepted." In the past year, studies have been published on the association between physical activity and breast cancer risk among Japanese [25], Chinese [26], 
Mexican [27], Tunisian [28], and US African American women [29]. A prospective study in Japan observed a $27 \%$ decrease risk of breast cancer (95\% CI, 0.54-1.00) comparing women whose recreational physical activity was $\geq 3$ days/week to women who reported $\leq 3$ days/month of physical activity [30]. One interesting result in this study is that the association was strongest among women with a BMI $\geq 25 \mathrm{~kg} / \mathrm{m}^{2}$ [30]. Similar results were observed in a cohort study of Chinese women in Shanghai, with a marked $27 \%$ reduction in breast cancer risk (95\% CI, 0.57-0.92) among postmenopausal women who expended at least 8 MET-hours/week/year compared to women who exercised < 8 MET-hours/week/year [26]. This association, too, was restricted to 'heavier' women, in this study, those with a BMI $\geq 23.73 \mathrm{~kg} / \mathrm{m}^{2}$. Both premenopausal and postmenopausal women in this Shanghai study showed increasing breast cancer risk with increasing lifetime occupational sitting time and decreasing occupation energy expenditure [26].

Several other case-control studies published in the past 18 months have some shortcoming (limited history of breast cancer, small sample size, hospital-based study design). A population-based case-control study of 1,000 incident breast cancer cases and 1,074 healthy controls in Mexico only considered physical activity in the previous 12 months, with minimal although statistically significant decreases in breast cancer risk among both premenopausal and postmenopausal women [27]. A small hospital-based case-control study in Tunisia assessed lifetime total physical activity (recreational, occupational and housework activity), showing marked reductions in postmenopausal, but not premenopausal breast cancer risk associated with lifetime physical activity [28]. Another case-control study with limited information on physical activity (past year) and small sample size, conducted among African American women in the Washington DC metropolitan area, observed $64 \%$ reduced risk (95\% CI, 0.17-0.75) among women who engaged in $\geq 2$ hours/week of vigorous physical activity (e.g., running, aerobics) in the past year compared to women who did not participate in any vigorous activity [29]. The study lacked sufficient statistical power to assess risk among premenopausal women, a common limitation of studies. Thus, the association was limited to postmenopausal women.

Risk specific to breast cancer subtypes defined by estrogen receptor (ER), progesterone receptor (PR) and HER-2/neu status of the tumor is of great interest. The Women's Health Initiative (WHI) observed decreases in breast cancer risk associated with recreational physical activity among postmenopausal women with ER-positive breast cancer [31•]. The highest tertile of moderate/low-intensity physical activity was associated with a $15 \%$ decrease in ER-positive breast cancer risk (HR=0.85; $95 \%$ CI, 0.74-0.98) [31•]. Similar results were observed for triple-negative breast cancer and for strenuous recreational physical activity; however, the findings were not statistically significant [31•]. A major limitation to this and previous studies stratifying by hormone receptor status is the inability to comprehensively classify triple negative breast cancer, due to missing HER2 status (unknown in $40 \%$ of cases in WHI study). Another limitation of the WHI study was its failure to break down the ER-positive subtype by PR and HER-2 status.

The Women's Contraceptive and Reproductive Experiences (CARE) Study investigated whether the use of menopausal hormone therapy modified the inverse association between recreational physical activity and breast cancer risk in postmenopausal women reported in a prior publication [32]. Similar risk reductions were observed among women who never used hormones, those who used hormones for less than 5 years and women who were currently taking estrogen only. Associations were not observed among other hormone use subgroups; however, despite the large size of this study, tests of homogeneity were not able to statistically demonstrate that hormone therapy modified the association between physical activity and breast cancer risk. 
As with colorectal cancer, an emphasis has been made on trying to detect risk factors earlier in the disease process. A report from the Nurses' Health Study II cohort showed that lifetime recreational physical activity was associated with risk of benign breast disease and columnar cell lesions, which may be precursors to breast cancer [33•]. Women who engaged in 3953.9 MET-hours/week (equivalent to 13 hours/week of walking or 3.25 hours/week of running) had substantially reduced risk of proliferative benign breast disease [Relative Risk $(\mathrm{RR})=0.63 ; 95 \% \mathrm{CI}, 0.33-1.19]$ [33•]. Although risk reduction was greater for women with low BMI at age 18 years, no statistical differences in RR could be demonstrated when comparing women with BMI $<21 \mathrm{~kg} / 2$ to those with higher BMI in late adolescence. These results suggest that the benefit of physical activity may be initiated early in the carcinogenic process and emphasize the importance of starting physical activity at young ages and maintaining physical activity throughout life.

\section{Breast Cancer Survival}

Breast cancer survivorship literature has shown evidence that pre-diagnosis and postdiagnosis physical activity reduce all-cause and breast cancer-specific mortality among breast cancer survivors [19•]. A recently published meta-analysis reported that pre-diagnosis physical activity was associated with lower all-cause mortality $(\mathrm{OR}=0.82,95 \% \mathrm{CI}, 0.67-$ 0.99 ), and that post-diagnosis activity was associated with reduced all-cause mortality $(\mathrm{OR}=0.59,95 \% \mathrm{CI}, 0.53-0.65)$, breast cancer-specific mortality (OR=0.66, $95 \% \mathrm{CI}, 0.57-$ $0.77)$ and disease recurrence $(\mathrm{OR}=0.76,95 \% \mathrm{CI}, 0.66-0.87)$ [34•]. These associations were modified by BMI and hormone receptor status. Pre-diagnosis physical activity was associated with lower breast cancer-specific mortality among women with a BMI $<25 \mathrm{~kg}$ / $\mathrm{m}^{2}$ (not overweight or obese), whereas post-diagnosis physical activity was associated with reductions only among women with a BMI $\geq 25 \mathrm{~kg} / \mathrm{m}^{2}$. Further, reductions in both breast cancer-specific and all-cause mortality were observed among women diagnosed with ERpositive, but not ER-negative, tumors. Chen et al. assessed associations between postdiagnosis recreational physical activity and survivorship among 4,826 women diagnosed with stages I-III breast cancer identified 6 months after diagnosis through the populationbased Shanghai Cancer Registry, and observed similar results [35]. Compared to women who did not participate in any recreational activity, reductions in breast cancer-specific mortality were observed for women who participated in regular physical activity ( $\geq 8.3$ MET-hours/week) within 18 months after diagnosis (HR=0.72, $95 \% \mathrm{CI}, 0.57-0.93)$ and within 36 months after diagnosis ( $\mathrm{HR}=0.59,95 \%$ CI, 0.45-0.76) [35]. Likewise, reductions in all-cause mortality were observed for women who participated in regular physical activity within 18 months after diagnosis (HR=0.65, $95 \% \mathrm{CI}, 0.51-0.83$ ) and within 36 months after diagnosis $(\mathrm{HR}=0.65,95 \% \mathrm{CI}, 0.51-0.84)$ [35]. Contrary to what was observed in the metaanalysis, Chen et al. observed reduced mortality only among women with ER-negative breast tumors $\left(\mathrm{HR}_{\text {all-cause }}=0.40,95 \% \mathrm{CI}, 0.29-0.59 ; \mathrm{HR}_{\text {breast cancer-specfic }}=0.36,95 \% \mathrm{CI}\right.$, $0.24-0.56)$ [35].

\section{Other Cancer Sites}

\section{Cancer Development}

Endometrial Cancer-The evidence showing that regular physical activity lowers endometrial cancer risk is accumulating, but is less convincing than that for breast or colon cancer [2]. A meta-analysis of prospective cohort studies considering the literature published through 2009 indicates that recreational physical activity lowers endometrial cancer risk by $27 \%$ (95\% CI, 0.58-0.93) and occupational activity lowers risk by (95\% CI, 0.71-0.88) [36•]. The authors further investigated sitting time in relation to endometrial cancer risk using data from the NIH-AARP Diet and Health Study, and observed that greater sitting time was independently associated with increased endometrial cancer risk [36•]. Two case- 
control studies found similar results [37, 38]. In one study, endometrial cancer risk was reduced $39 \%$ (95\% CI, 0.43-0.87) among women with the highest level of lifetime, combined, occupational and recreational activity ( 291.9 MET-hours/week) when compared to women with the least activity; moderate intensity lifetime activity alone also resulted in a substantial risk reduction [37]. Activity performed at younger and older adult ages produced similar risk reductions. Further, the inverse associations were strongest in obese and overweight women. Arem et al. also observed that moderate-intensity to vigorous-intensity sports/recreational physical activity was associated with a $34 \%$ reduction in endometrial cancer risk (95\% CI, 0.50-0.87) comparing active to inactive women ( 27.5 MET-hours/ week versus 0 MET-hours/week); however, in contrast with the John et al. study [37], the associations were stronger among women with BMI < 25 [38]. Further, risk was greater among women sitting more than 8 hours/day than among women sitting less than 4 hours/ day $(\mathrm{OR}=1.52,95 \% \mathrm{CI}, 1.07-2.16)$.

Prostate Cancer-The evidence for an association between physical activity and prostate cancer has been classified as probable [2]. In a 2002 review of the literature, Friedenreich and Orenstein suggested that prostate cancer risk is reduced 10-30\% when comparing the most active men to the least active men [4]. An update to this review, based on 22 additional studies, indicates that the majority of recent research studies observed protective effects [39]. Leitzmann and Rohrmann added that the associations with reduced risk may be most apparent for fatal prostate cancer [40] A current systematic review and meta-analysis, including 19 cohort and 24 case-control studies, agrees [41•]. A pooled $19 \%$ reduction in risk (95\% CI, 0.73-0.91) was observed for occupational physical activity, and a $5 \%$ reduction (95\% CI, 0.89-1.00) was observed for recreational physical activity, comparing the most physically active men to the least active. An issue that somewhat reduces our confidence in these estimates is that considerable heterogeneity between studies was observed. Further analysis of this compilation of studies suggested that only physical activity performed between the ages of 20 and 65 years was associated with prostate cancer risk [41•].

Lung Cancer-Physical activity may reduce lung cancer risk 30-40\% [4], but the literature is not convincing, as one cannot ignore potential residual confounding or effect modification due to smoking. Recent studies have attempted to address this issue by estimating risk within subgroups defined by smoking status. A recent review suggests an inverse relationship between heavy lifetime physical activity and lung cancer in former and current smokers that is consistent across all histologies, but is not observed not among those who have never smoked [6•]. A small case-control study of current and former smokers enrolled in the Cologne Smoking Study came to a similar conclusion, observing a lower risk of lung cancer among participants who were physically active compared to those who were not $(\mathrm{OR}=0.53,95 \% \mathrm{CI}, 0.29-0.97)$ [42]. Due to sex differences in lung cancer pathology, risk factors and prognosis, current research has also begun to investigate the association for men and women separately [43]. The recent literature consists of small case-control studies [44] that suffer from an inability to examine risk in subgroups by histology, smoking status or sex (reduced statistical power), and a survival bias in that rapidly fatal cases or those who are too ill to be interviewed are excluded from the study population.

Ovarian Cancer-Existing literature on risk of ovarian cancer in relation to physical activity is inconclusive [2]. A previous meta-analysis of 12 studies observed a $21 \%$ reduction in ovarian cancer risk $(95 \% \mathrm{CI}, 0.70-0.85)$ for case-control studies and a $19 \%$ reduction in risk (95\% CI, 0.57-1.17) in cohort studies, when comparing the women who performed the most recreational physical activity to those performing the least amount [45]. Three additional studies showed no association [46-48], and the most recent study observed 
a nonsignificant 10-20\% reduction in ovarian cancer risk for those who engaged in one or more hours/week of recreational aerobic activity [49].

Renal Cell Carcinoma-Physical activity has been studied in relation to renal cell carcinoma, in part because of the known deleterious effects of high body mass index and hypertension on the risk of renal cell cancer; however, no association has been established. A review of genitourinary cancers observed significant protective effects in 8 of 15 studies of physical activity in relation to renal cell carcinoma, with an average $8 \%$ reduction in risk comparing individuals with the highest level of physical activity to those with the lowest level of activity [50•]. Reductions in risk were greater for recreational than for other forms of activity and for activity performed later in life.

Pancreatic Cancer-A meta-analysis of 28 studies of pancreas cancer showed total lifetime physical activity and occupational activity were associated with reduced risk [51]. Non-significant reductions in risk were observed for recreational physical activity and transportation (walking and cycling as a form of commuting). Significant heterogeneity was present across the studies, making it difficult to find a definitive answer. An analysis of a prospective study conducted in the Netherlands indicated that risk of pancreatic cancer was reduced $20 \%$ (95\% CI, 0.64-0.99) among those who ever versus never participated in a sports activity [52]. Pancreatic cancer was not associated with physical activity among individuals whose past participation in sports activity was $<2$ hours/week (versus those with no activity (HR=1.18, $95 \% \mathrm{CI}, 0.78-1.79)$, whereas risk of pancreatic cancer was reduced among those whose participation in sports activity ranged from 2 to $<4$ hours/week $(\mathrm{HR}=0.67,95 \% \mathrm{CI}, 0.47-0.96)$ or was $\geq 4$ hours/week $(\mathrm{HR}=0.76,95 \% \mathrm{CI}, 0.57-1.00 ; P$ trend $=0.05$ ) [52]. No associations were noted for other recreational activities or occupational activity.

Non-Hodgkin's Lymphoma (NHL)—Recently, three studies have investigated the association between NHL and physical activity. The Women's Health Initiative (WHI) combined clinical trial and observational cohort study observed statistically non-significant positive associations between total recreational physical activity (MET-hours/week), as well as hours of moderate or strenuous recreational physical activity per week, and risk of NHL; however, no specific subtype associations were observed [53]. The European Prospective Investigation into Cancer and Nutrition (EPIC) found no evidence that total physical activity influenced the risk of NHL or B-cell NHL, overall or by subtype, for either men or for women [54]. Similarly, the American Cancer Society Cancer Prevention Study-II Cohort observed no association [55]. However, women who reported sitting at least six hours/day (versus those reporting sitting for fewer than 3 hours/day) had increased risk of multiple myeloma $(\mathrm{HR}=2.40,95 \% \mathrm{CI}, 1.45-3.97)$.

\section{Other Cancer Sites Survival}

Evidence that physical activity reduces cancer patients' mortality is beginning to accumulate for prostate and lung cancers. Results of one study indicate that post-diagnosis physical activity is associated with a 46-49 \% reduction in all-cause mortality among prostate cancer survivors and a $61 \%$ reduction in prostate cancer-specific mortality [56]. Further, among prostate cancer patients, Richman et al. found those who engaged in at least 3 hours of brisk walking per week had the lowest risk of disease progression [57]. The latest lung cancer literature has focused on increasing cardiorespiratory fitness [58]. Sui et al. observed an inverse association between cardiorespiratory fitness and lung cancer-specific mortality among current and former smoking men, and a 12-fold increase in risk of death among current smokers with low cardiorespiratory fitness level [58]. Moorman et al. observed modest reductions in ovarian cancer mortality among non-obese women who reported 
engaging in at least 2 hours of recreational physical activity per week one year prior to cancer diagnosis [49].

\section{Conclusion}

The evidence of a beneficial effect of physical activity on cancer risk is accumulating rapidly and can be classified as "convincing" for colon and breast cancer and "probable" or "possible" for several other cancer sites. To increase our understanding of these associations, it will be necessary to conduct studies that are optimally designed, collect detailed lifetime histories of physical activity, and examine the effects across different population subgroups. By considering the potential, underlying biological mechanisms that are operative, it should be possible to refine study designs, questionnaires, and biomarker measures to enhance our understanding of the causal pathways that define the relationships between physical activity and cancer incidence, and to make our public health recommendations regarding physical activity more specific. Although it is clear that recommendations for appropriate changes in physical activity levels are important public health messages, we still have no exact physical activity prescriptions to give the public generally for all cancers and specifically for individual cancers. Further, we still need to be able to address the following questions: What are the ages when physical activity provides its greatest benefit? What types of activity will provide the greatest protection against cancer or enhance survival? What activity patterns (intensity, frequency, duration of activity) are optimal? Can enhancements of the built environment facilitate participation in physical activity?

\section{Acknowledgments}

The work reported here was supported by National Cancer Institute grants K05 CA136967 and R01 CA77398.

\section{References and Recommended Reading}

Papers of particular interest, published recently, have been highlighted as:

- Of importance

1. National Center for Chronic Disease Prevention and Health Promotion. Physical Activity and Health: A Report of the Surgeon General. National Center for Chronic Disease Prevention and Health Promotion, Atlanta, GA: 1996.

2. Vainio, H.; Bianchini, F., editors. IARC handbooks of cancer prevention Weight control and physical activity. Vol. Vol. 6. Lyon, France: IARC Press; 2000.

3. Friedenreich CM. Physical activity and cancer prevention: from observational to intervention research. Cancer Epidemiol Biomarkers Prev. 2001; 10(4):287-301. [PubMed: 11319168]

4. Friedenreich CM, Orenstein MR. Physical activity and cancer prevention: etiologic evidence and biological mechanisms. J Nutr. 2002; 132(11 Suppl):3456S-3464S. [PubMed: 12421870]

5. Ainsworth BE, Haskell WL, Whitt MC, et al. Compendium of physical activities: an update of activity codes and MET intensities. Med Sci Sports Exerc. 2000; 32(9 Suppl):S498-S504. [PubMed: 10993420]

6. Anzuini F, Battistella A, Izzotti A. Physical activity and cancer prevention: a review of current evidence and biological mechanisms. J Prev Med Hyg. 2011; 52(4):174-180. [PubMed: 22442921] This review summarizes potential biological mechanisms mediating the association between physical activity and cancer, and highlights evidence from both animal models and human epidemiological studies.

7. Harriss DJ, Atkinson G, Batterham A, et al. Lifestyle factors and colorectal cancer risk (2): a systematic review and meta-analysis of associations with leisure-time physical activity. Colorectal Dis. 2009; 11(7):689-701. [PubMed: 19207713] 
8. Spence RR, Heesch KC, Brown WJ. A systematic review of the association between physical activity and colorectal cancer risk. Scand J Med Sci Sports. 2009; 19(6):764-781. [PubMed: 19705997]

9. Friedenreich CM, Neilson HK, Lynch BM. State of the epidemiological evidence on physical activity and cancer prevention. Eur J Cancer. 2010; 46(14):2593-2604. [PubMed: 20843488]

10. Wolin KY, Yan Y, Colditz GA, Lee IM. Physical activity and colon cancer prevention: a metaanalysis. Br J Cancer. 2009; 100(4):611-616. [PubMed: 19209175]

11. Iacopetta B. Are there two sides to colorectal cancer? Int J Cancer. 2002; 101(5):403-408. [PubMed: 12216066]

12. Boyle T, Heyworth J, Bull F, et al. Timing and intensity of recreational physical activity and the risk of subsite-specific colorectal cancer. Cancer Causes Control. 2011; 22(12):1647-1658. [PubMed: 21922204] This case-control study examines the timing and intensity of recreational physical activity on subsite-specific colorectal cancer risk, showing reductions in distal not proximal colon cancer risk associated with vigorous physical activity. Further, this is one of the few studies to observe an association with rectal cancer.

13. Owen N, Healy GN, Matthews CE, Dunstan DW. Too much sitting: the population health science of sedentary behavior. Exerc Sport Sci Rev. 2010; 38(3):105-113. [PubMed: 20577058]

14. Boyle T, Fritschi L, Heyworth J, Bull F. Long-term sedentary work and the risk of subsite-specific colorectal cancer. Am J Epidemiol. 2011; 173(10):1183-1191. [PubMed: 21421743]

15. Hughes LA, Simons CC, van den Brandt PA, et al. Body size and colorectal cancer risk after 16.3 years of follow-up: an analysis from the Netherlands Cohort Study. Am J Epidemiol. 2011; 174(10):1127-1139. [PubMed: 21984660]

16. Markowitz SD, Bertagnolli MM. Molecular origins of cancer: Molecular basis of colorectal cancer. N Engl J Med. 2009; 361(25):2449-2460. [PubMed: 20018966]

17. Hughes LA, Simons CC, van den Brandt PA, et al. Body size, physical activity and risk of colorectal cancer with or without the CpG island methylator phenotype (CIMP). PLoS One. 2011; 6(4):e18571. [PubMed: 21483668]

18. Wolin KY, Yan Y, Colditz GA. Physical activity and risk of colon adenoma: a meta-analysis. Br J Cancer. 2011; 104(5):882-885. [PubMed: 21304525] This systematic literature review and metaanalysis examines physical activity/exercise and risk/prevalence of colon adenomas or polyps, which are known precursor lesions of colon cancer, and reports a significant inverse association between physical activity and colon adenomas, suggesting that physical activity can have an important role in preventing lesions that may lead to colon cancer.

19. Ballard-Barbash R, Friedenreich CM, Courneya KS, et al. Physical activity, biomarkers, and disease outcomes in cancer survivors: a systematic review. J Natl Cancer Inst. 2012; 104(11):815840. [PubMed: 22570317] In this article, the authors systematically review studies that examined relationships between physical activity and mortality (cancer-specific and all-cause) and/or cancer biomarkers, concluding consistent evidence exists for reduced all-cause, breast cancer-specific, and colon cancer-specific mortality evidence for other cancers is insufficient, and exercise may result in beneficial changes in the circulating level of insulin, insulin-related pathways, inflammation and possibly, immunity.

20. Davies NJ, Batehup L, Thomas R. The role of diet and physical activity in breast, colorectal, and prostate cancer survivorship: a review of the literature. Br J Cancer. 2011; 105(Suppl 1):S52-S73. [PubMed: 22048034] This review summarizes observational studies examining the role of diet and physical activity in breast, colorectal, and prostate cancer survivorship, stating substantial evidence that physical activity can help relieve treatment-related symptoms, such as fatigue, exists, as well as promising data suggesting that physical activity after diagnosis is associated with improved survival. The article briefly describes some of the proposed biological mechanisms including the role of adiposity, metabolic and sex hormones, growth factors, immunological processes and chronic inflammation.

21. Morikawa T, Kuchiba A, Yamauchi M, et al. Association of CTNNB1 (beta-catenin) alterations, body mass index, and physical activity with survival in patients with colorectal cancer. JAMA. 2011; 305(16):1685-1694. [PubMed: 21521850]

22. Bernstein L, Henderson BE, Hanisch R, et al. Physical exercise and reduced risk of breast cancer in young women. J Natl Cancer Inst. 1994; 86(18):1403-1408. [PubMed: 8072034] 
23. Carpenter CL, Ross RK, Paganini-Hill A, Bernstein L. Lifetime exercise activity and breast cancer risk among post-menopausal women. Br J Cancer. 1999; 80(11):1852-1858. [PubMed: 10468309]

24. Carpenter CL, Ross RK, Paganini-Hill A, Bernstein L. Effect of family history, obesity and exercise on breast cancer risk among postmenopausal women. Int J Cancer. 2003; 106(1):96-102. [PubMed: 12794763]

25. Iwasaki M, Tsugane S. Risk factors for breast cancer: epidemiological evidence from Japanese studies. Cancer Sci. 2011; 102(9):1607-1614. [PubMed: 21624009]

26. Pronk A, Ji BT, Shu XO, et al. Physical activity and breast cancer risk in Chinese women. Br J Cancer. 2011; 105(9):1443-1450. [PubMed: 21934685]

27. Sanchez-Zamorano LM, Flores-Luna L, Angeles-Llerenas A, et al. Healthy lifestyle on the risk of breast cancer. Cancer Epidemiol Biomarkers Prev. 2011; 20(5):912-922. [PubMed: 21335508]

28. Awatef M, Olfa G, Rim C, et al. Physical activity reduces breast cancer risk: a case-control study in Tunisia. Cancer Epidemiol. 2011; 35(6):540-544. [PubMed: 21470932]

29. Sheppard VB, Makambi K, Taylor T, et al. Physical activity reduces breast cancer risk in African American women. Ethn Dis. 2011; 21(4):406-411. [PubMed: 22428342]

30. Suzuki R, Iwasaki M, Yamamoto S, et al. Leisure-time physical activity and breast cancer risk defined by estrogen and progesterone receptor status - the Japan Public Health Center-based Prospective Study. Prev Med. 2011; 52(3-4):227-233. [PubMed: 21295062]

31. Phipps AI, Chlebowski RT, Prentice R, et al. Body size, physical activity, and risk of triplenegative and estrogen receptor-positive breast cancer. Cancer Epidemiol Biomarkers Prev. 2011; 20(3):454-463. [PubMed: 21364029] Using data from 155,723 women enrolled in the Women's Health Initiative clinical trial and observational cohort, this article assesses associations between body size, recreational physical activity, and risk of triple-negative and estrogen receptor-positive $(\mathrm{ER}+)$ breast cancers, observing a reduction in risk associated with moderate/low-intensity physical activity for ER+ tumors only.

32. Dieli-Conwright CM, Sullivan-Halley J, Patel A, et al. Does hormone therapy counter the beneficial effects of physical activity on breast cancer risk in postmenopausal women? Cancer Causes Control. 2011; 22(3):515-522. [PubMed: 21213036]

33. Jung MM, Colditz GA, Collins LC, et al. Lifetime physical activity and the incidence of proliferative benign breast disease. Cancer Causes Control. 2011; 22(9):1297-1305. [PubMed: 21748307] This study of 40,318 women enrolled in the Nurses' Health Study II cohort is the first to prospectively examine the association between recreational physical activity and the development of premalignant breast lesions, and suggests that exercise may be inversely associated with the risk of developing proliferative benign breast disease.

34. Ibrahim EM, Al-Homaidh A. Physical activity and survival after breast cancer diagnosis: metaanalysis of published studies. Med Oncol. 2011; 28(3):753-765. [PubMed: 20411366] This metaanalysis examines studies of associations between pre-diagnosis and post-diagnosis physical activity and breast cancer outcomes, and provides evidence for inverse relationships for reduced all-cause mortality, breast cancer-specific mortality, and disease recurrence.

35. Chen X, Lu W, Zheng W, et al. Exercise after diagnosis of breast cancer in association with survival. Cancer Prev Res (Phila). 2011; 4(9):1409-1418. [PubMed: 21795422]

36. Moore SC, Gierach GL, Schatzkin A, Matthews CE. Physical activity, sedentary behaviours, and the prevention of endometrial cancer. Br J Cancer. 2010; 103(7):933-938. [PubMed: 20877336]

The authors of this article performed a meta-analysis of studies examining physical activity, sitting time and endometrial cancer, as well as investigated sitting time in relation to endometrial cancer risk using data from the NIH-AARP Diet and Health Study, and conclude that the totality of evidence convincingly indicates that physical activity reduces risk.

37. John EM, Koo J, Horn-Ross PL. Lifetime physical activity and risk of endometrial cancer. Cancer Epidemiol Biomarkers Prev. 2010; 19(5):1276-1283. [PubMed: 20406960]

38. Arem H, Irwin ML, Zhou Y, et al. Physical activity and endometrial cancer in a population-based case-control study. Cancer Causes Control. 2011; 22(2):219-226. [PubMed: 21110224]

39. Young-McCaughan S. Potential for prostate cancer prevention through physical activity. World J Urol. 2012; 30(2):167-179. [PubMed: 22198724] 
40. Leitzmann MF, Rohrmann S. Risk factors for the onset of prostatic cancer: age, location, and behavioral correlates. Clin Epidemiol. 2012; 4:1-11. [PubMed: 22291478]

41. Liu Y, Hu F, Li D, et al. Does physical activity reduce the risk of prostate cancer? A systematic review and meta-analysis. Eur Urol. 2011; 60(5):1029-1044. [PubMed: 21802197] This systematic review and meta-analysis summarizes results from 19 cohort and 24 case-control studies, examining the association between physical activity and prostate cancer and suggests a small inverse association between occupational, recreational and total physical activity and prostate cancer risk.

42. Schmidt A, Jung J, Ernstmann N, et al. The association between active participation in a sports club, physical activity and social network on the development of lung cancer in smokers: a casecontrol study. BMC Res Notes. 2012; 5:2. [PubMed: 22214387]

43. Tardon A, Lee WJ, Delgado-Rodriguez M, et al. Leisure-time physical activity and lung cancer: a meta-analysis. Cancer Causes Control. 2005; 16(4):389-397. [PubMed: 15953981]

44. Lin Y, Cai L. Environmental and dietary factors and lung cancer risk among chinese women: a case-control study in southeast china. Nutr Cancer. 2012; 64(4):508-514. [PubMed: 22489989]

45. Olsen CM, Bain CJ, Jordan SJ, et al. Recreational physical activity and epithelial ovarian cancer: a case-control study, systematic review, and meta-analysis. Cancer Epidemiol Biomarkers Prev. 2007; 16(11):2321-2330. [PubMed: 18006921]

46. Weiderpass E, Margolis KL, Sandin S, et al. Prospective study of physical activity in different periods of life and the risk of ovarian cancer. Int J Cancer. 2006; 118(12):3153-3160. [PubMed: 16425259]

47. Lahmann PH, Friedenreich C, Schulz M, et al. Physical activity and ovarian cancer risk: the European Prospective Investigation into Cancer and Nutrition. Cancer Epidemiol Biomarkers Prev. 2009; 18(1):351-354. [PubMed: 19124520]

48. Leitzmann MF, Koebnick C, Moore SC, et al. Prospective study of physical activity and the risk of ovarian cancer. Cancer Causes Control. 2009; 20(5):765-773. [PubMed: 19116765]

49. Moorman PG, Jones LW, Akushevich L, Schildkraut JM. Recreational physical activity and ovarian cancer risk and survival. Ann Epidemiol. 2011; 21(3):178-187. [PubMed: 21296269]

50. Leitzmann MF. Physical activity and genitourinary cancer prevention. Recent Results Cancer Res. 2011; 186:43-71. [PubMed: 21113760] This article reviews epidemiologic evidence of associations between physical activity and genitourinary cancers (prostate, bladder, renal cell, and testicular cancers) and suggests a weak inverse relationship between physical activity prostate and renal cell cancer risk.

51. O'Rorke MA, Cantwell MM, Cardwell CR, et al. Can physical activity modulate pancreatic cancer risk? a systematic review and meta-analysis. Int J Cancer. 2010; 126(12):2957-2968. [PubMed: 19856317]

52. Heinen MM, Verhage BA, Goldbohm RA, et al. Physical activity, energy restriction, and the risk of pancreatic cancer: a prospective study in the Netherlands. Am J Clin Nutr. 2011; 94(5):13141323. [PubMed: 21955648]

53. Kabat GC, Kim MY, Jean Wactawski W, et al. Anthropometric factors, physical activity, and risk of non-Hodgkin's lymphoma in the Women's Health Initiative. Cancer Epidemiol. 2012; 36(1):5259. [PubMed: 21816698]

54. van Veldhoven CM, Khan AE, Teucher B, et al. Physical activity and lymphoid neoplasms in the European Prospective Investigation into Cancer and nutrition (EPIC). Eur J Cancer. 2011; 47(5): 748-760. [PubMed: 21159506]

55. Teras LR, Gapstur SM, Diver WR, et al. Recreational physical activity, leisure sitting time and risk of non-Hodgkin lymphoid neoplasms in the American Cancer Society Cancer Prevention Study II Cohort. Int J Cancer. 2012

56. Kenfield SA, Stampfer MJ, Giovannucci E, Chan JM. Physical activity and survival after prostate cancer diagnosis in the health professionals follow-up study. J Clin Oncol. 2011; 29(6):726-732. [PubMed: 21205749]

57. Richman EL, Kenfield SA, Stampfer MJ, et al. Physical activity after diagnosis and risk of prostate cancer progression: data from the cancer of the prostate strategic urologic research endeavor.

Cancer Res. 2011; 71(11):3889-3895. [PubMed: 21610110] 
58. Sui X, Lee DC, Matthews CE, et al. Influence of cardiorespiratory fitness on lung cancer mortality. Med Sci Sports Exerc. 2010; 42(5):872-878. [PubMed: 19996990] 